\title{
Energy Stable Schemes for Cahn-Hilliard Phase-Field Model of Two-Phase Incompressible Flows***
}

\author{
Jie SHEN* Xiaofeng YANG ${ }^{* *}$ \\ (Dedicated to Professor Roger Temam on the Occasion of his 70 th Birthday)
}

\begin{abstract}
Numerical approximations of Cahn-Hilliard phase-field model for the two-phase incompressible flows are considered in this paper. Several efficient and energy stable time discretization schemes for the coupled nonlinear Cahn-Hilliard phase-field system for both the matched density case and the variable density case are constructed, and are shown to satisfy discrete energy laws which are analogous to the continuous energy laws.
\end{abstract}

Keywords Phase-field, Two-phase flow, Navier-Stokes, Cahn-Hilliard, Energy stable 2000 MR Subject Classification 65M12, 65M70, 65P99, 65Z05, 76T99

\section{Introduction}

The phase-field approach for multi-phase incompressible flows have attracted much attention recently (cf. $[11,2,16,12,15,26]$ and the references therein). Since the phase-field (or diffusive interface) model can be considered as an approximation to the sharp interface model, one can use the gradient flow based on either the conserved Cahn-Hilliard dynamics (cf. [5]) or the Allen-Cahn dynamics (cf. [1]) with a non-local Lagrange multiplier, leading to the CahnHilliard phase-field model and Allen-Cahn phase-field model, respectively. Both models, at least in the matched density case, can be derived from an energetic variational approach. Thus, they admit an energy law, making it possible to design numerical schemes which satisfy a corresponding discrete energy law that automatically ensures their numerical stability (cf., for instance, $[7,14,3,25])$.

However, most of the analysis and simulation of the phase-field model for two-phase flows have been restricted to the matched density case or with a Boussinesq approximation. The main difficulty for two-phase flows with different density is that the standard phase-field model with variable density does not admit an energy law, making it difficult to carry out mathematical and numerical analysis. In a recent work (cf. [23]), the authors proposed a phase-field model with variable density which admits an energy law, and constructed efficient and simple energy stable time discretization schemes for the corresponding Allen-Cahn phase-field model.

\footnotetext{
Manuscript received on July 6, 2010. Published online August 25, 2010.

*Department of Mathematics, Purdue University, West Lafayette, IN, 47907, USA.

E-mail: shen@math.purdue.edu

** Department of Mathematics, University of South Carolina, Columbia, SC, 29208, USA.

E-mail: xfyang@math.sc.edu

*** Project supported by the National Science Foundation (No. DMS-0915066)
} 
The main objective of this paper is to construct efficient and simple energy stable time discretization schemes for the Cahn-Hilliard phase-field model with matched density and variable density. The main additional theoretical and numerical difficulty associated with the CahnHilliard model, as opposed to the Allen-Cahn model, is that the fourth-order spatial derivatives are involved in the Cahn-Hilliard equation for the phase function. By using a mixed formulation for the fourth-order Cahn-Hilliard phase equation and using the chemical potential to reformulate the surface tension term in the momentum equation, we are able to extend the results presented in [23] for the Allen-Cahn phase-field model to the Cahn-Hilliard phase-field model.

The rest of the paper is organized as follows. In the next section, we present the CahnHilliard phase-field model for two-phase incompressible flows with matched density and variable density. Then, in Section 3, we construct several efficient time discretization schemes for both matched density and variable density cases, and show that they are unconditionally energy stable. Some numerical results and discussions are presented in the last section.

\section{Cahn-Hilliard Phase-Field Model}

We consider a mixture of two immiscible, incompressible fluids in a confined domain $\Omega$ with densities $\rho_{1}, \rho_{2}$ and viscosities $\mu_{1}, \mu_{2}$, respectively. To fix the idea, we assume $0<\rho_{1} \leq \rho_{2}$ and viscosities $0<\mu_{1} \leq \mu_{2}$. In order to identify the regions occupied by the two fluids, we introduce a phase function $\phi$ such that

$$
\phi(x, t)= \begin{cases}1 & \text { fluid } 1 \\ -1 & \text { fluid } 2\end{cases}
$$

with a thin smooth transition layer of thickness $\eta$ connecting the two fluids so the interface of the mixture can be described by $\Gamma_{t}=\{x: \phi(x, t)=0\}$. Let $F(\phi)=\frac{1}{4 \eta^{2}}\left(\phi^{2}-1\right)^{2}$ be the Ginzburg-Landau double-well potential. Define the mixing energy functional as

$$
W(\phi, \nabla \phi)=\int_{\Omega}\left(\frac{1}{2}|\nabla \phi|^{2}+F(\phi)\right) \mathrm{d} x
$$

which represents the competition between the hydro-philic and hydro-phobic properties of the two-phase flow. We can then determine the dynamics of the phase function $\phi$ by the following Cahn-Hilliard gradient flow (cf. [5, 11, 2, 16, 12, 15]):

$$
\begin{aligned}
& \phi_{t}+(u \cdot \nabla) \phi+\gamma \Delta w=0, \\
& w=\frac{\delta W}{\delta \phi}=\Delta \phi-f(\phi),
\end{aligned}
$$

where $w$ is the so called chemical potential and $\gamma$ is a mobility constant related to the relaxation time scale and $f(\phi)=F^{\prime}(\phi)$.

On the other hand, the momentum equation for the two-phase system takes the usual form:

$$
\rho\left(u_{t}+(u \cdot \nabla) u\right)=\nabla \cdot \tau
$$

where the total stress $\tau=\mu D(u)-p I+\tau_{e}$ with $D(u)=\nabla u+\nabla u^{\mathrm{T}}$ and $\tau_{e}$ is the extra elastic stress induced by the interfacial surface tension. It can be shown, using the least-action-principle 
and the mixing energy functional defined above, that the momentum equation becomes:

$$
\rho\left(u_{t}+(u \cdot \nabla) u\right)=\nabla \cdot(\mu D(u)-p I-\lambda(\nabla \phi \otimes \nabla \phi)),
$$

where $\lambda$ is the mixing energy density.

In the above, $\rho$ and $\mu$ are slave variables defined by the linear average

$$
\rho(\phi)=\frac{\rho_{1}-\rho_{2}}{2} \phi+\frac{\rho_{1}+\rho_{2}}{2}, \quad \mu(\phi)=\frac{\mu_{1}-\mu_{2}}{2} \phi+\frac{\mu_{1}+\mu_{2}}{2} .
$$

To fix the idea, we consider the boundary conditions

$$
\left.u\right|_{\partial \Omega}=0,\left.\quad \frac{\partial \phi}{\partial n}\right|_{\partial \Omega}=0,\left.\quad \frac{\partial w}{\partial n}\right|_{\partial \Omega}=0 .
$$

Hence, the Cahn-Hilliard phase equation (2.3) and the momentum equations (2.5) with the boundary condition (2.7), together with the incompressibility constraint

$$
\nabla \cdot u=0
$$

form a complete system for $(u, p, \phi, w)$ with $\rho$ and $\mu$ given by (2.6).

In the case of matched density, namely $\rho_{1}=\rho_{2}=\rho$, it is well-known that the Cahn-Hilliard phase-field system (2.3)-(2.8)-(2.5) admits the following energy law (cf., for instance, [15]):

$$
\frac{\mathrm{d}}{\mathrm{d} t} \int_{\Omega}\left(\frac{1}{2} \rho|u|^{2}+\frac{\lambda}{2}|\nabla \phi|^{2}+\lambda F(\phi)\right) \mathrm{d} x=-\int_{\Omega}\left(\frac{\mu}{2}|D(u)|^{2}+\lambda \gamma|\nabla w|^{2}\right) \mathrm{d} x .
$$

However, for the case of variable density, namely, $\rho_{1} \neq \rho_{2}$, the above no longer holds. Therefore, it is proposed in [23] to replace (2.5) by the modified momentum equation

$$
\sigma(\sigma u)_{t}+(\rho u \cdot \nabla) u+\frac{1}{2} \nabla \cdot(\rho u) u-\nabla \cdot \mu D(u)+\nabla p+\lambda \nabla \cdot(\nabla \phi \otimes \nabla \phi)=0,
$$

where $\sigma=\sqrt{\rho}$. Note that (2.5) and (2.10) are identical if we assume that the density $\rho$ satisfies the mass conservation

$$
\rho_{t}+\nabla \cdot(\rho u)=0
$$

which is obviously true if $\rho$ is a constant, but do not necessarily hold near the interface if $\rho_{1} \neq \rho_{2}$ and $\rho$ is determined by $(2.6)$.

The main advantage of the new formulation is that the modified Cahn-Hilliard phase-field system (2.3)-(2.8)-(2.10) now admits the following energy law (cf. [23]):

$$
\frac{\mathrm{d}}{\mathrm{d} t} \int_{\Omega}\left(\frac{1}{2}|\sigma u|^{2}+\frac{\lambda}{2}|\nabla \phi|^{2}+\lambda F(\phi)\right) \mathrm{d} x=-\int_{\Omega}\left(\frac{\mu}{2}|D(u)|^{2}+\lambda \gamma|\nabla w|^{2}\right) \mathrm{d} x .
$$

\section{Energy Stable Time Discretizations and Their Stability Analysis}

In this section, we study time discretizations of the new phase-field model introduced in the last section. The goal is to construct time discretization schemes which satisfy a discrete energy law similar to the continuous cases (2.9) and (2.12) and are easy to solve in practice.

In [23], we have studied a phase-field model with the Allen-Cahn phase equation instead of the Cahn-Hilliard phase equation in (2.3), and constructed several energy stable numerical 
schemes. In this paper, we shall consider the time discretization of the Cahn-Hilliard phase-field system with matched density (2.3)-(2.8)-(2.5) and with different density (2.3)-(2.8)-(2.10).

Let us first reformulate these systems into equivalent forms which are more convenient for numerical approximation.

While the Cahn-Hilliard equation does not satisfy the maximum principle, it has been shown in [4] that for a truncated potential $F(\phi)$ with quadratic growth at infinities, the maximum norm of the solution for the Cahn-Hilliard equation is bounded. Therefore, it has been a common practice to consider the Cahn-Hilliard equation with a truncated double-well potential $F(\phi)$ (cf. $[13,6]$ ). Since we expect that $\phi$ takes value in the interval $[-1,1]$, we can replace $F(\phi)$ by

$$
F(\phi)= \begin{cases}\frac{1}{\eta^{2}}(\phi-1)^{2}, & \phi>1, \\ \frac{1}{4 \eta^{2}}\left(\phi^{2}-1\right)^{2}, & \phi \in[-1,1], \\ \frac{1}{\eta^{2}}(\phi+1)^{2}, & \phi<-1 .\end{cases}
$$

Then, setting $f(\phi)=F^{\prime}(\phi)$, we have

$$
\max _{\phi \in \mathbb{R}}\left|f^{\prime}(\phi)\right| \leq \frac{2}{\eta^{2}}
$$

Accordingly, we set

$$
\widehat{\phi}= \begin{cases}\phi, & |\phi| \leq 1, \\ \operatorname{sign}(\phi), & |\phi|>1,\end{cases}
$$

and use it to update the variable density and viscosity by

$$
\rho(\phi)=\frac{\rho_{1}-\rho_{2}}{2} \widehat{\phi}+\frac{\rho_{1}+\rho_{2}}{2}, \quad \mu(\phi)=\frac{\mu_{1}-\mu_{2}}{2} \widehat{\phi}+\frac{\mu_{1}+\mu_{2}}{2} .
$$

It is clear that $\rho$ and $\mu$ as defined above are such that $0<\rho_{1} \leq \rho(\phi) \leq \rho_{2}$ and $0<\mu_{1} \leq \mu(\phi) \leq$ $\mu_{2}$.

Next, we use the identity

$$
\nabla \cdot(\nabla \phi \otimes \nabla \phi)=\Delta \phi \nabla \phi+\frac{1}{2} \nabla|\nabla \phi|^{2},
$$

and to derive

$$
\nabla p+\lambda \nabla \cdot(\nabla \phi \otimes \nabla \phi)=\nabla\left(p+\frac{1}{2} \lambda|\nabla \phi|^{2}+\lambda F(\phi)\right)+\lambda w \nabla \phi .
$$

Therefore, if we define the modified pressure as $\widetilde{p}=p+\frac{1}{2} \lambda|\nabla \phi|^{2}+\lambda F(\phi)$ and still denote it by $p$ for simplicity, we can rewrite the system (2.3)-(2.8)-(2.5) for the matched density case (assuming $\rho_{1}=\rho_{2}=1$ for simplicity) as

$$
\begin{aligned}
& \phi_{t}+u \cdot \nabla \phi+\gamma \Delta w=0, \\
& w-\Delta \phi+f(\phi)=0, \\
& u_{t}+(u \cdot \nabla) u-\nabla \cdot \mu D(u)+\nabla p+\lambda w \nabla \phi=0, \\
& \nabla \cdot u=0,
\end{aligned}
$$


with $\mu$ given by (3.4). Similarly, the system (2.3)-(2.8)-(2.10) for the variable density case can be rewritten as

$$
\begin{aligned}
& \phi_{t}+u \cdot \nabla \phi=-\gamma \Delta w, \\
& w=\Delta \phi-f(\phi), \\
& \sigma(\sigma u)_{t}+(\rho u \cdot \nabla) u+\frac{1}{2} \nabla \cdot(\rho u) u-\nabla \cdot \mu D(u)+\nabla p+\lambda w \nabla \phi=0, \\
& \nabla \cdot u=0
\end{aligned}
$$

where $\sigma=\sqrt{\rho}$ with $\rho$ and $\mu$ are given by (3.4).

In this section, we design numerical algorithms which admit an energy law and overcome three main difficulties associated with this coupled nonlinear system, namely, (i) the nonlinear coupling of the velocity, pressure and the phase function; (ii) the stiffness associated with the interfacial width $\eta$; (iii) the pressure solver with large density ratio.

\subsection{The case of matched density}

In this case, it is relatively easy to construct an efficient, energy stable scheme. The following is a first-order scheme for the system (3.7) based on the pressure-correction projection method (cf. $[8,22]$ ) with a stabilized treatment for the Cahn-Hilliard phase equation (cf. [24]).

Given initial conditions $u^{0}$ and $\phi^{0}$, we compute $\left(\phi^{n+1}, w^{n+1}, \widetilde{u}^{n+1}, u^{n+1}, p^{n+1}\right)$ for $n \geq 0$ by

$$
\begin{gathered}
\frac{1}{\delta t}\left(\phi^{n+1}-\phi^{n}\right)+\left(\widetilde{u}^{n+1} \cdot \nabla\right) \phi^{n}+\gamma \Delta w^{n+1}=0, \\
w^{n+1}+\frac{1}{\eta^{2}}\left(\phi^{n+1}-\phi^{n}\right)-\Delta \phi^{n+1}+f\left(\phi^{n}\right)=0, \\
\left.\partial_{n} \phi^{n+1}\right|_{\partial \Omega}=0,\left.\quad \partial_{n} w^{n+1}\right|_{\partial \Omega}=0 ; \\
\left\{\begin{array}{l}
\frac{\widetilde{u}^{n+1}-u^{n}}{\delta t}+\left(u^{n} \cdot \nabla\right) \widetilde{u}^{n+1}-\nabla \cdot \mu^{n} D\left(\widetilde{u}^{n+1}\right)+\nabla p^{n}+\lambda w^{n+1} \nabla \phi^{n}=0, \\
\left.\widetilde{u}^{n+1}\right|_{\partial \Omega}=0
\end{array}\right.
\end{gathered}
$$

with $\mu^{n}=\frac{\mu_{1}-\mu_{2}}{2} \widehat{\phi}^{n}+\frac{\mu_{1}+\mu_{2}}{2}$; and

$$
\left\{\begin{array}{l}
\frac{u^{n+1}-\widetilde{u}^{n+1}}{\delta t}+\nabla\left(p^{n+1}-p^{n}\right)=0, \\
\nabla \cdot u^{n+1}=0 \\
\left.n \cdot u^{n+1}\right|_{\partial \Omega}=0 .
\end{array}\right.
$$

Remark 3.1 As in [24], a stabilizing term $\frac{1}{\eta^{2}}\left(\phi^{n+1}-\phi^{n}\right)$ is introduced in the second equation of (3.9a). This term introduces an additional consistency error of order $\frac{\gamma \delta t}{\eta^{2}} \phi^{\prime}(\xi)$ which is of the same order as the error introduced by the explicit treatment of $f(\phi)$.

It is also clear that a second-order version can be easily constructed.

Remark 3.2 At each time step, the above scheme involves a weakly coupled linear system for $\left(\phi^{n+1}, w^{n+1}, \widetilde{u}^{n+1}\right)$. In fact, if we replace $\widetilde{u}^{n+1}$ by $\widetilde{u}^{n}$ in $(3.9 \mathrm{a})$, then $\left(\phi^{n+1}, w^{n+1}\right)$ and $\widetilde{u}^{n+1}$ are decoupled and can be obtained separately by solving two elliptic equations/systems. 
We show below that the above scheme admits a discrete energy law.

Theorem 3.1 The scheme (3.9) is unconditionally stable and satisfies the following discrete energy law:

$$
\begin{aligned}
& \left\|u^{n+1}\right\|_{L^{2}}^{2}+\delta t^{2}\left\|\nabla p^{n+1}\right\|_{L^{2}}^{2}+\lambda\left\|\nabla \phi^{n+1}\right\|_{L^{2}}^{2}+2 \lambda\left(F\left(\phi^{n+1}\right), 1\right)+\mu_{1} \delta t\left\|D\left(\widetilde{u}^{n+1}\right)\right\|_{L^{2}}^{2} \\
\leq & \left\|u^{n}\right\|_{L^{2}}^{2}+\delta t^{2}\left\|\nabla p^{n}\right\|_{L^{2}}^{2}+\lambda\left\|\nabla \phi^{n}\right\|_{L^{2}}^{2}+2 \lambda\left(F\left(\phi^{n}\right), 1\right) .
\end{aligned}
$$

Proof First of all, notice that

$$
2 \delta t\left(\mu^{n} D\left(\widetilde{u}^{n+1}\right), \nabla \widetilde{u}^{n+1}\right)=\delta t\left\|\sqrt{\mu^{n}} D\left(\widetilde{u}^{n+1}\right)\right\|_{L^{2}}^{2} \geq \mu_{1} \delta t\left\|D\left(\widetilde{u}^{n+1}\right)\right\|_{L^{2}}^{2} .
$$

Hence, taking the inner product of $(3.9 \mathrm{~d})$ with $2 \delta t \widetilde{u}^{n+1}$, we derive

$$
\begin{aligned}
& \left\|\widetilde{u}^{n+1}\right\|_{L^{2}}^{2}-\left\|u^{n}\right\|_{L^{2}}^{2}+\left\|\widetilde{u}^{n+1}-u^{n}\right\|_{L^{2}}^{2}+\mu_{1} \delta t\left\|D\left(\widetilde{u}^{n+1}\right)\right\|_{L^{2}}^{2} \\
& +2 \lambda \delta t\left(w^{n+1} \nabla \phi^{n}, \widetilde{u}^{n+1}\right) \leq 0 .
\end{aligned}
$$

Taking the inner product of (3.9e) with $2 \delta t \nabla p^{n}$, we obtain

$$
\delta t^{2}\left(\left\|\nabla p^{n+1}\right\|_{L^{2}}^{2}-\left\|\nabla p^{n}\right\|_{L^{2}}^{2}-\left\|\nabla p^{n+1}-\nabla p^{n}\right\|_{L^{2}}^{2}\right)=2 \delta t\left(\widetilde{u}^{n+1}, \nabla p^{n}\right) .
$$

On the other hand, we derive from $(3.9 \mathrm{e})$ that

$$
\delta t^{2}\left\|\nabla p^{n+1}-\nabla p^{n}\right\|_{L^{2}}^{2}=\left\|\widetilde{u}^{n+1}-u^{n+1}\right\|_{L^{2}}^{2}
$$

and

$$
\left\|u^{n+1}\right\|_{L^{2}}^{2}+\left\|u^{n+1}-\widetilde{u}^{n+1}\right\|_{L^{2}}^{2}=\left\|\widetilde{u}^{n+1}\right\|_{L^{2}}^{2} .
$$

Combining the above inequalities, we find

$$
\begin{aligned}
& \left\|u^{n+1}\right\|_{L^{2}}^{2}-\left\|u^{n}\right\|_{L^{2}}^{2}+\left\|\widetilde{u}^{n+1}-u^{n}\right\|_{L^{2}}^{2}+\mu_{1} \delta t\left\|D\left(\widetilde{u}^{n+1}\right)\right\|_{L^{2}}^{2} \\
& +\delta t^{2}\left(\left\|\nabla p^{n+1}\right\|_{L^{2}}^{2}-\left\|\nabla p^{n}\right\|_{L^{2}}^{2}\right)+2 \lambda \delta t\left(w^{n+1} \nabla \phi^{n}, \widetilde{u}^{n+1}\right) \leq 0 .
\end{aligned}
$$

Next, taking the inner product of $(3.9 \mathrm{a})$ with $-2 \lambda \delta t w^{n+1}$, we have

$$
-2 \lambda\left(\phi^{n+1}-\phi^{n}, w^{n+1}\right)-2 \lambda \delta t\left(\widetilde{u}^{n+1} \nabla \phi^{n}, w^{n+1}\right)+2 \lambda \gamma \delta t\left\|\nabla w^{n+1}\right\|_{L^{2}}^{2}=0,
$$

and taking the inner product of $(3.9 \mathrm{~b})$ with $2 \lambda\left(\phi^{n+1}-\phi^{n}\right)$,

$$
\begin{aligned}
& 2 \lambda\left(w^{n+1}, \phi^{n+1}-\phi^{n}\right)+\frac{2 \lambda}{\eta^{2}}\left\|\phi^{n+1}-\phi^{n}\right\|_{L^{2}}^{2} \\
& +\lambda\left(\left\|\nabla \phi^{n+1}\right\|_{L^{2}}^{2}-\left\|\nabla \phi^{n}\right\|_{L^{2}}^{2}+\left\|\nabla \phi^{n+1}-\nabla \phi^{n}\right\|_{L^{2}}^{2}\right)+2 \lambda\left(f\left(\phi^{n}\right), \phi^{n+1}-\phi^{n}\right)=0 .
\end{aligned}
$$

For the last term in (3.17), we use the Taylor expansion

$$
F\left(\phi^{n+1}\right)-F\left(\phi^{n}\right)=f\left(\phi^{n}\right)\left(\phi^{n+1}-\phi^{n}\right)+\frac{f^{\prime}\left(\xi^{n}\right)}{2}\left(\phi^{n+1}-\phi^{n}\right)^{2} .
$$


Combining (3.15), (3.16), (3.17) and (3.18), we obtain

$$
\begin{aligned}
& \left\|u^{n+1}\right\|_{L^{2}}^{2}-\left\|u^{n}\right\|_{L^{2}}^{2}+\left\|\widetilde{u}^{n+1}-u^{n}\right\|_{L^{2}}^{2}+\mu_{1} \delta t\left\|D\left(\widetilde{u}^{n+1}\right)\right\|_{L^{2}}^{2} \\
& +\delta t^{2}\left(\left\|\nabla p^{n+1}\right\|_{L^{2}}^{2}-\left\|\nabla p^{n}\right\|_{L^{2}}^{2}\right)+2 \lambda \gamma \delta t\left\|\nabla w^{n+1}\right\|_{L^{2}}^{2}+\frac{2 \lambda}{\eta^{2}}\left\|\phi^{n+1}-\phi^{n}\right\|_{L^{2}}^{2} \\
& +\lambda\left(\left\|\nabla \phi^{n+1}\right\|_{L^{2}}^{2}-\left\|\nabla \phi^{n}\right\|_{L^{2}}^{2}+\left\|\nabla \phi^{n+1}-\nabla \phi^{n}\right\|_{L^{2}}^{2}+2 \lambda\left(F\left(\phi^{n+1}\right)-F\left(\phi^{n}\right), 1\right)\right. \\
\leq & \lambda\left(f^{\prime}\left(\xi^{n}\right)\left(\phi^{n+1}-\phi^{n}\right), \phi^{n+1}-\phi^{n}\right) \leq \frac{2 \lambda}{\eta^{2}}\left\|\phi^{n+1}-\phi^{n}\right\|_{L^{2}}^{2} .
\end{aligned}
$$

We can then conclude from the above inequality.

\subsection{The case of variable density}

In this case, there are at least two additional difficulties when compared with the matched density case. First, it is not easy to construct a second-order scheme based on a pressurecorrection formulation (cf. [9]). Second, the subsystem to determine a pressure approximation if often an elliptic equation with $\frac{1}{\rho}$ as variable coefficient, making it difficult to solve when the density ratio is large.

We shall first construct first and second-order schemes based on a gauge-Uzawa formulation (cf. $[17,19,24])$. These schemes require solving a pressure elliptic equation with $\frac{1}{\rho}$ as variable coefficient. Then, we construct schemes based on a pressure-stabilized formulation (cf. [20, 22, $8,10]$ ) which only require solving a pressure Poisson equation.

\subsubsection{Schemes based on a gauge-Uzawa formulation}

We construct below a first-order gauge-Uzawa scheme for the phase-field model (3.8).

Given initial conditions $\phi^{0}, s^{0}=0, u^{0}$. We compute $\left(\phi^{n+1}, \widetilde{u}^{n+1}, u^{n+1}, s^{n+1}\right)$ for $n \geq 0$ by

$$
\begin{gathered}
\frac{1}{\delta t}\left(\phi^{n+1}-\phi^{n}\right)+\left(\widetilde{u}^{n+1} \cdot \nabla\right) \phi^{n}+\gamma \Delta w^{n+1}=0, \\
w^{n+1}+\frac{1}{\eta^{2}}\left(\phi^{n+1}-\phi^{n}\right)-\Delta \phi^{n+1}+f\left(\phi^{n}\right)=0, \\
\left.\partial_{n} \phi^{n+1}\right|_{\partial \Omega}=0,\left.\quad \partial_{n} w^{n+1}\right|_{\partial \Omega}=0 ; \\
\sigma^{n+1} \frac{\sigma^{n+1} \widetilde{u}^{n+1}-\sigma^{n} u^{n}}{\delta t}+\rho^{n}\left(u^{n} \cdot \nabla\right) \widetilde{u}^{n+1}+\frac{1}{2}\left(\nabla \cdot\left(\rho^{n} u^{n}\right)\right) \widetilde{u}^{n+1} \\
-\nabla \cdot \mu^{n+1} D\left(\widetilde{u}^{n+1}\right)+\mu_{1} \nabla s^{n}+\lambda w^{n+1} \nabla \phi^{n}=0, \\
\left.\widetilde{u}^{n+1}\right|_{\partial \Omega}=0
\end{gathered}
$$

with

$$
\rho^{n+1}=\frac{\rho_{1}-\rho_{2}}{2} \widehat{\phi}^{n+1}+\frac{\rho_{1}+\rho_{2}}{2}, \quad \mu^{n+1}=\frac{\mu_{1}-\mu_{2}}{2} \widehat{\phi}^{n+1}+\frac{\mu_{1}+\mu_{2}}{2}, \quad \sigma^{n+1}=\sqrt{\rho^{n+1}} ;
$$

and

$$
\begin{aligned}
& -\nabla \cdot\left(\frac{1}{\rho^{n+1}} \nabla \psi^{n+1}\right)=\nabla \cdot \widetilde{u}^{n+1}, \\
& \left.\partial_{n} \psi^{n+1}\right|_{\partial \Omega}=0
\end{aligned}
$$




$$
\begin{aligned}
& u^{n+1}=\widetilde{u}^{n+1}+\frac{1}{\rho^{n+1}} \nabla \psi^{n+1}, \\
& s^{n+1}=s^{n}-\nabla \cdot \widetilde{u}^{n+1} .
\end{aligned}
$$

Remark 3.3 In the above scheme, $s^{n}$ is the so called gauge variable, and a proper pressure approximation is [19]:

$$
p^{n+1}=-\frac{\psi^{n+1}}{\delta t}+\mu_{1} s^{n+1}
$$

Remark 3.4 Similar to (3.9), the above scheme involves a weakly coupled system for $\left(\phi^{n+1}, w^{n+1}, \widetilde{u}^{n+1}\right)$ and an elliptic equation with density as variable coefficient for the pseudopressure " $\psi^{n+1} "$.

We have the following result for the scheme (3.19).

Theorem 3.2 The scheme (3.19) is unconditionally stable and satisfies the following discrete energy law:

$$
\begin{aligned}
& \left\|\sigma^{n+1} \widetilde{u}^{n+1}\right\|_{L^{2}}^{2}+\mu_{1} \delta t\left\|s^{n+1}\right\|_{L^{2}}^{2}+\lambda\left\|\nabla \phi^{n+1}\right\|_{L^{2}}^{2}+2 \lambda\left(F\left(\phi^{n+1}\right), 1\right) \\
& +\delta t\left(2 \lambda \gamma\left\|\nabla w^{n+1}\right\|_{L^{2}}^{2}+\mu_{1}\left\|\nabla \widetilde{u}^{n+1}\right\|_{L^{2}}^{2}\right) \\
\leq & \left\|\sigma^{n} \widetilde{u}^{n}\right\|_{L^{2}}^{2}+\mu_{1} \delta t\left\|s^{n}\right\|_{L^{2}}^{2}+\lambda\left\|\nabla \phi^{n}\right\|_{L^{2}}^{2}+2 \lambda\left(F\left(\phi^{n}\right), 1\right) .
\end{aligned}
$$

Proof Taking the inner product of $(3.19 \mathrm{~b})$ with $2 \delta t \widetilde{u}^{n+1}$, using $(3.10)$, we obtain

$$
\begin{aligned}
& \left\|\sigma^{n+1} \widetilde{u}^{n+1}\right\|_{L^{2}}^{2}-\left\|\sigma^{n} u^{n}\right\|_{L^{2}}^{2}+\left\|\sigma^{n+1} \widetilde{u}^{n+1}-\sigma^{n} u^{n}\right\|_{L^{2}}^{2}+\mu_{1} \delta t\left\|D\left(\widetilde{u}^{n+1}\right)\right\|_{L^{2}}^{2} \\
& +2 \mu_{1} \delta t\left(\nabla s^{n}, \widetilde{u}^{n+1}\right)+2 \lambda \delta t\left(w^{n+1} \nabla \phi^{n}, \widetilde{u}^{n+1}\right) \leq 0 .
\end{aligned}
$$

Using (3.19d) and (3.19e), we obtain

$$
\begin{aligned}
\left(\sigma^{n} u^{n}, \sigma^{n} u^{n}\right) & =\left(\rho^{n} u^{n}, u^{n}\right)=\left(\rho^{n}\left(\widetilde{u}^{n}+\frac{1}{\rho^{n}} \nabla \psi^{n}\right), u^{n}\right)=\left(\rho^{n} \widetilde{u}^{n}, u^{n}\right) \\
& =\left(\rho^{n} \widetilde{u}^{n}, \widetilde{u}^{n}+\frac{1}{\rho^{n}} \nabla \psi^{n}\right)=\left\|\sigma^{n} \widetilde{u}^{n}\right\|_{L^{2}}^{2}+\left(u^{n}-\frac{1}{\rho^{n}} \nabla \psi^{n}, \nabla \psi^{n}\right) \\
& =\left\|\sigma^{n} \widetilde{u}^{n}\right\|_{L^{2}}^{2}-\left\|\frac{1}{\sigma^{n}}\right\|_{L^{2}}^{2},
\end{aligned}
$$

and

$$
\begin{aligned}
2 \mu_{1} \delta t\left(\nabla s^{n}, \widetilde{u}^{n+1}\right) & =2 \mu_{1} \delta t\left(s^{n},-\nabla \cdot \widetilde{u}^{n+1}\right)=2 \mu_{1} \delta t\left(s^{n}, s^{n+1}-s^{n}\right) \\
& =\mu_{1} \delta t\left(\left\|s^{n+1}\right\|_{L^{2}}^{2}-\left\|s^{n}\right\|_{L^{2}}^{2}-\left\|s^{n+1}-s^{n}\right\|_{L^{2}}^{2}\right) \\
& =\mu_{1} \delta t\left(\left\|s^{n+1}\right\|_{L^{2}}^{2}-\left\|s^{n}\right\|_{L^{2}}^{2}\right)-\mu_{1} \delta t\left\|\nabla \cdot \widetilde{u}^{n+1}\right\|_{L^{2}}^{2}
\end{aligned}
$$

It is easy to check by integration by parts that

$$
\|D(u)\|_{L^{2}}^{2}=\|\nabla u\|_{L^{2}}^{2}+\|\nabla \cdot u\|_{L^{2}}^{2}, \quad \forall u \in H_{0}^{1}(\Omega)^{d} .
$$

Hence, we have

$$
\mu_{1}\left\|\nabla \cdot \widetilde{u}^{n+1}\right\|_{L^{2}}^{2}+\mu_{1}\left\|\nabla \widetilde{u}^{n+1}\right\|_{L^{2}}^{2}=\left\|\sqrt{\mu_{1}} D\left(\widetilde{u}^{n+1}\right)\right\|_{L^{2}}^{2} \leq\left\|\sqrt{\mu^{n+1}} D\left(\widetilde{u}^{n+1}\right)\right\|_{L^{2}}^{2} .
$$


Combining the above inequalities, we find

$$
\begin{aligned}
& \left\|\sigma^{n+1} \widetilde{u}^{n+1}\right\|_{L^{2}}^{2}-\left\|\sigma^{n} \widetilde{u}^{n}\right\|_{L^{2}}^{2}+\left\|\frac{1}{\sigma^{n}}\right\|_{L^{2}}^{2}+\left\|\sigma^{n+1} \widetilde{u}^{n+1}-\sigma^{n} u^{n}\right\|_{L^{2}}^{2} \\
& +\mu_{1} \delta t\left(\left\|s^{n+1}\right\|_{L^{2}}^{2}-\left\|s^{n}\right\|_{L^{2}}^{2}\right)+\delta t \mu_{1}\left\|\nabla \widetilde{u}^{n+1}\right\|_{L^{2}}^{2}+2 \lambda \delta t\left(w^{n+1} \nabla \phi^{n}, \widetilde{u}^{n+1}\right) \leq 0 .
\end{aligned}
$$

Taking the inner product of $(3.19 \mathrm{a})$ with $-2 \lambda \delta t w^{n+1},(3.19 \mathrm{~b})$ with $2 \lambda\left(\phi^{n+1}-\phi^{n}\right)$ and using the same procedure as in the proof of Theorem 3.1, we obtain

$$
\begin{aligned}
& \left\|\sigma^{n+1} \widetilde{u}^{n+1}\right\|_{L^{2}}^{2}-\left\|\sigma^{n} \widetilde{u}^{n}\right\|_{L^{2}}^{2}+\left\|\frac{1}{\sigma^{n}} \nabla \psi^{n}\right\|^{2}+\left\|\sigma^{n+1} \widetilde{u}^{n+1}-\sigma^{n} u^{n}\right\|_{L^{2}}^{2}+\delta t \mu_{1}\left\|\nabla \widetilde{u}^{n+1}\right\|_{L^{2}}^{2} \\
& +\mu_{1} \delta t\left(\left\|s^{n+1}\right\|_{L^{2}}^{2}-\left\|s^{n}\right\|_{L^{2}}^{2}\right)+2 \lambda \delta t \gamma\left\|\nabla w^{n+1}\right\|_{L^{2}}^{2} \\
& +\lambda\left(\left\|\nabla \phi^{n+1}\right\|_{L^{2}}^{2}-\left\|\nabla \phi^{n}\right\|_{L^{2}}^{2}+\left\|\nabla \phi^{n+1}-\nabla \phi^{n}\right\|_{L^{2}}^{2}\right) \\
& +2 \lambda\left(F\left(\phi^{n+1}\right)-F\left(\phi^{n}\right), 1\right) \leq 0 .
\end{aligned}
$$

We can then conclude from the above inequality.

Remark 3.5 A formally second-order scheme can be constructed by combining the secondorder scheme in [19] and the approach for the phase equation in (3.19) as follows:

For the sake of simplicity, we shall denote, for any sequence $\left\{a^{k}\right\}, a^{*, k+1}=2 a^{k}-a^{k-1}$.

$$
\begin{aligned}
& \frac{3 \phi^{n+1}-4 \phi^{n}+\phi^{n-1}}{2 \delta t}+\left(\widetilde{u}^{n+1} \cdot \nabla\right) \phi^{*, n+1}+\gamma \Delta w^{n+1}=0, \\
& w^{n+1}+\frac{1}{\eta^{2}}\left(\phi^{n+1}-2 \phi^{n}+\phi^{n-1}\right)-\Delta \phi^{n+1}+2 f\left(\phi^{n}\right)-f\left(\phi^{n-1}\right)=0, \\
& \left.\partial_{n} \phi^{n+1}\right|_{\partial \Omega}=0,\left.\quad \partial_{n} w^{n+1}\right|_{\partial \Omega}=0 ; \\
& \rho^{n+1} \frac{3 \widetilde{u}^{n+1}-4 u^{n}+u^{n-1}}{2 \delta t}+\rho^{n+1}\left(u^{*, n+1} \cdot \nabla\right) \widetilde{u}^{n+1}+\frac{1}{2}\left(\nabla \cdot\left(\rho^{n+1} u^{*, n+1}\right)\right) \widetilde{u}^{n+1} \\
& -\nabla \cdot \mu^{n+1} D\left(\widetilde{u}^{n+1}\right)+\nabla p^{n}+\mu_{1} \nabla s^{n}+\lambda w^{n+1} \nabla \phi^{n}=0, \\
& \left.\widetilde{u}^{n+1}\right|_{\partial \Omega}=0
\end{aligned}
$$

with

$$
\begin{gathered}
\rho^{n+1}=\frac{\rho_{1}-\rho_{2}}{2} \widehat{\phi}^{n+1}+\frac{\rho_{1}+\rho_{2}}{2}, \quad \mu^{n+1}=\frac{\mu_{1}-\mu_{2}}{2} \widehat{\phi}^{n+1}+\frac{\mu_{1}+\mu_{2}}{2} ; \\
-\nabla \cdot\left(\frac{1}{\rho^{n+1}} \nabla \psi^{n+1}\right)=\nabla \cdot \widetilde{u}^{n+1} \\
\left.\partial_{n} \psi^{n+1}\right|_{\partial \Omega}=0 ;
\end{gathered}
$$

and

$$
\begin{aligned}
& u^{n+1}=\widetilde{u}^{n+1}+\frac{1}{\rho^{n+1}} \nabla \psi^{n+1}, \\
& s^{n+1}=s^{n}-\nabla \cdot \widetilde{u}^{n+1}, \\
& p^{n+1}=p^{n}-\frac{3}{2 \delta t} \psi^{n+1}+\bar{\mu} s^{n+1} .
\end{aligned}
$$




\subsubsection{Schemes based on a pressure-stabilization formulation}

We now construct schemes based on the pressure-stabilization formulation (cf., for instance, $[20,22,18,8])$, namely, the divergence free condition is replaced by

$$
\nabla \cdot u-\delta \Delta p_{t}=0
$$

where $\delta$ is a small parameter.

Inspired by the schemes presented in [10,23], we propose the following first-order scheme.

Given initial conditions $\phi^{0}, p^{0}=0, u^{0}$. We compute $\left(\phi^{n+1}, u^{n+1}, p^{n+1}\right)$ for $n \geq 0$ by

$$
\begin{aligned}
& \frac{\phi^{n+1}-\phi^{n}}{\delta t}+\left(u^{n+1} \cdot \nabla\right) \phi^{n}+\gamma \Delta w^{n+1}=0, \\
& w^{n+1}+\frac{1}{\eta^{2}}\left(\phi^{n+1}-\phi^{n}\right)-\Delta \phi^{n+1}+f\left(\phi^{n}\right)=0, \\
& \left.\partial_{n} \phi^{n+1}\right|_{\partial \Omega}=0,\left.\quad \partial_{n} w^{n+1}\right|_{\partial \Omega}=0 ;
\end{aligned}
$$

and

$$
\begin{aligned}
& \frac{\frac{1}{2}\left(\rho^{n+1}+\rho^{n}\right) u^{n+1}-\rho^{n} u^{n}}{\delta t}+\rho^{n}\left(u^{n} \cdot \nabla\right) u^{n+1}+\frac{1}{2}\left(\nabla \cdot\left(\rho^{n} u^{n}\right)\right) u^{n+1} \\
& -\nabla \cdot \mu^{n+1} D\left(u^{n+1}\right)+\nabla\left(2 p^{n}-p^{n-1}\right)+\lambda w^{n+1} \nabla \phi^{n}=0, \\
& \left.u^{n+1}\right|_{\partial \Omega}=0
\end{aligned}
$$

with

$$
\rho^{n+1}=\frac{\rho_{1}-\rho_{2}}{2} \widehat{\phi}^{n+1}+\frac{\rho_{1}+\rho_{2}}{2}, \quad \mu^{n+1}=\frac{\mu_{1}-\mu_{2}}{2} \widehat{\phi}^{n+1}+\frac{\mu_{1}+\mu_{2}}{2} ;
$$

and

$$
\begin{aligned}
& \Delta\left(p^{n+1}-p^{n}\right)=\frac{\rho_{1}}{\delta t} \nabla \cdot u^{n+1}, \\
& \left.\partial_{n} p^{n+1}\right|_{\partial \Omega}=0 .
\end{aligned}
$$

Remark 3.6 The above scheme involves a weakly coupled system for $\left(\phi^{n+1}, w^{n+1}, u^{n+1}\right)$ and a Poisson equation for the pressure increment. So this scheme is computationally more efficient than (3.19), particularly when the density ratio is large. On the other hand, the above scheme does not lead to a divergence-free approximation while (3.19) does.

Theorem 3.3 The scheme (3.28) is unconditionally stable and satisfies the following discrete energy law:

$$
\begin{aligned}
& \left\|\sigma^{n+1} u^{n+1}\right\|_{L^{2}}^{2}+\frac{\delta t^{2}}{\rho_{1}}\left\|\nabla p^{n+1}\right\|_{L^{2}}^{2}+\lambda\left\|\nabla \phi^{n+1}\right\|_{L^{2}}^{2}+2 \lambda\left(F\left(\phi^{n+1}\right), 1\right) \\
& +\delta t\left(2 \lambda \gamma\left\|\nabla w^{n+1}\right\|_{L^{2}}^{2}+\left\|\sqrt{\mu^{n+1}} D\left(u^{n+1}\right)\right\|_{L^{2}}^{2}\right) \\
\leq & \left\|\sigma^{n} u^{n}\right\|_{L^{2}}^{2}+\frac{\delta t^{2}}{\rho_{1}}\left\|\nabla p^{n}\right\|_{L^{2}}^{2}+\lambda\left\|\nabla \phi^{n}\right\|_{L^{2}}^{2}+2 \lambda\left(F\left(\phi^{n}\right), 1\right) .
\end{aligned}
$$

Proof Taking the inner product of (3.28d) with $2 \delta t u^{n+1}$, using the identity

$$
\begin{aligned}
\left(\frac{1}{2}\left(\rho^{n+1}+\rho^{n}\right) u^{n+1}-\rho^{n} u^{n}, 2 u^{n+1}\right)= & \left\|\sigma^{n+1} u^{n+1}\right\|_{L^{2}}^{2}-\left\|\sigma^{n} u^{n}\right\|_{L^{2}}^{2} \\
& +\left\|\sigma^{n}\left(u^{n+1}-u^{n}\right)\right\|_{L^{2}}^{2}
\end{aligned}
$$


and (3.10), we derive

$$
\begin{aligned}
& \left\|\sigma^{n+1} u^{n+1}\right\|_{L^{2}}^{2}-\left\|\sigma^{n} u^{n}\right\|_{L^{2}}^{2}+\left\|\sigma^{n}\left(u^{n+1}-u^{n}\right)\right\|_{L^{2}}^{2}+\delta t\left\|\sqrt{\mu^{n+1}} D\left(u^{n+1}\right)\right\|_{L^{2}}^{2} \\
& +2 \delta t\left(p^{n+1}-2 p^{n}+p^{n-1}, \nabla \cdot u^{n+1}\right) \\
& -2 \delta t\left(p^{n+1}, \nabla \cdot u^{n+1}\right)+2 \lambda \delta t\left(w^{n+1} \nabla \phi^{n}, u^{n+1}\right)=0 .
\end{aligned}
$$

Taking the inner product of $(3.28 \mathrm{e})$ with $\frac{2 \delta t^{2}}{\rho_{1}}\left(p^{n+1}-2 p^{n}+p^{n-1}\right)$ and with $-\frac{2 \delta t^{2}}{\rho_{1}} p^{n+1}$ separately, we obtain

$$
\begin{aligned}
& -\frac{\delta t^{2}}{\rho_{1}}\left(\left\|\nabla\left(p^{n+1}-p^{n}\right)\right\|_{L^{2}}^{2}-\left\|\nabla\left(p^{n}-p^{n-1}\right)\right\|_{L^{2}}^{2}+\left\|\nabla\left(p^{n+1}-2 p^{n}+p^{n-1}\right)\right\|_{L^{2}}^{2}\right) \\
= & 2 \delta t\left(\nabla \cdot u^{n+1}, p^{n+1}-2 p^{n}+p^{n-1}\right),
\end{aligned}
$$

and

$$
\frac{\delta t^{2}}{\rho_{1}}\left(\left\|\nabla p^{n+1}\right\|_{L^{2}}^{2}-\left\|\nabla p^{n}\right\|_{L^{2}}^{2}+\| \nabla\left(p^{n+1}-p^{n} \|_{L^{2}}^{2}\right)=-2 \delta t\left(\nabla \cdot u^{n+1}, p^{n+1}\right) .\right.
$$

Adding the above two equalities together, we have

$$
\begin{aligned}
& 2 \delta t\left(p^{n+1}-2 p^{n}+p^{n-1}, \nabla \cdot u^{n+1}\right)-2 \delta t\left(p^{n+1}, \nabla \cdot u^{n+1}\right) \\
= & \frac{\delta t^{2}}{\rho_{1}}\left(\left\|\nabla p^{n+1}\right\|_{L^{2}}^{2}-\left\|\nabla p^{n}\right\|_{L^{2}}^{2}\right)+\frac{\delta t^{2}}{\rho_{1}}\left\|\nabla\left(p^{n}-p^{n-1}\right)\right\|_{L^{2}}^{2} \\
& -\frac{\delta t^{2}}{\rho_{1}}\left\|\nabla\left(p^{n+1}-2 p^{n}+p^{n-1}\right)\right\|_{L^{2}}^{2} .
\end{aligned}
$$

Taking the difference of (3.28e) at step $n+1$ and step $n$, we derive

$$
\frac{\delta t^{2}}{\rho_{1}}\left\|\nabla\left(p^{n+1}-2 p^{n}+p^{n-1}\right)\right\|_{L^{2}}^{2} \leq \rho_{1}\left\|u^{n+1}-u^{n}\right\|_{L^{2}}^{2} \leq\left\|\sigma^{n}\left(u^{n+1}-u^{n}\right)\right\|_{L^{2}}^{2} .
$$

Combining the above inequalities together, we derive

$$
\begin{aligned}
& \left\|\sigma^{n+1} u^{n+1}\right\|_{L^{2}}^{2}-\left\|\sigma^{n} u^{n}\right\|_{L^{2}}^{2}+\delta t\left\|\sqrt{\mu^{n+1}} D\left(u^{n+1}\right)\right\|_{L^{2}}^{2} \\
& +\frac{\delta t^{2}}{\rho_{1}}\left(\left\|\nabla p^{n+1}\right\|_{L^{2}}^{2}-\left\|\nabla p^{n}\right\|_{L^{2}}^{2}\right)+\frac{\delta t^{2}}{\rho_{1}}\left\|\nabla\left(p^{n+1}-p^{n}\right)\right\|_{L^{2}}^{2} \\
& +2 \lambda \delta t\left(w^{n+1} \nabla \phi^{n}, u^{n+1}\right) \leq 0 .
\end{aligned}
$$

Finally, taking the inner product of (3.28a) with $-2 \lambda \delta t w^{n+1},(3.28 \mathrm{~b})$ with $2 \lambda\left(\phi^{n+1}-\phi^{n}\right)$ and using the same procedure as in the proof of Theorem 3.1, we obtain

$$
\begin{aligned}
& \left\|\sigma^{n+1} u^{n+1}\right\|_{L^{2}}^{2}-\left\|\sigma^{n} u^{n}\right\|_{L^{2}}^{2}+\delta t\left\|\sqrt{\mu^{n+1}} D\left(u^{n+1}\right)\right\|_{L^{2}}^{2} \\
& +\frac{\delta t^{2}}{\rho_{1}}\left(\left\|\nabla p^{n+1}\right\|_{L^{2}}^{2}-\left\|\nabla p^{n}\right\|_{L^{2}}^{2}\right)+\frac{\delta t^{2}}{\rho_{1}}\left\|\nabla\left(p^{n+1}-p^{n}\right)\right\|_{L^{2}}^{2} \\
& +2 \lambda \gamma \delta t\left\|\nabla w^{n+1}\right\|_{L^{2}}^{2}+\lambda\left(\left\|\nabla \phi^{n+1}\right\|_{L^{2}}^{2}-\left\|\nabla \phi^{n}\right\|_{L^{2}}^{2}+\left\|\nabla \phi^{n+1}-\nabla \phi^{n}\right\|_{L^{2}}^{2}\right) \\
& +2 \lambda\left(F\left(\phi^{n+1}\right)-F\left(\phi^{n}\right), 1\right) \leq 0 .
\end{aligned}
$$

Remark 3.7 We can construct a second-order version of the scheme (3.28) by combining the approaches for the phase equation in (3.28) and for the velocity-pressure in [10]. As before, 
we still denote, for any sequence $\left\{a^{k}\right\}, a^{*, k+1}=2 a^{k}-a^{k-1}$. Then, a second-order version of (3.28) reads

$$
\begin{aligned}
& \frac{3 \phi^{n+1}-4 \phi^{n}+\phi^{n-1}}{2 \delta t}+\left(u^{n+1} \cdot \nabla\right) \phi^{*, n+1}+\gamma \Delta w^{n+1}=0, \\
& w^{n+1}+\frac{1}{\eta^{2}}\left(\phi^{n+1}-2 \phi^{n}+\phi^{n-1}\right)-\Delta \phi^{n+1}+2 f\left(\phi^{n}\right)-f\left(\phi^{n-1}\right)=0, \\
& \left.\partial_{n} \phi^{n+1}\right|_{\partial \Omega}=0,\left.\quad \partial_{n} w^{n+1}\right|_{\partial \Omega}=0 ; \\
& \frac{\rho^{n+1}}{2 \delta t}\left(3 u^{n+1}-4 u^{n}+u^{n-1}\right)+\rho^{n+1}\left(\nabla \cdot u^{*, n+1}\right) u^{n+1}-\nabla \cdot \mu^{n+1} D\left(u^{n+1}\right) \\
& +\nabla\left(p^{n}+\frac{4}{3} \psi^{n}-\frac{1}{3} \psi^{n-1}\right)+\lambda w^{n+1} \nabla \phi^{n}=0, \\
& \left.u^{n+1}\right|_{\partial \Omega}=0
\end{aligned}
$$

with

$$
\rho^{n+1}=\frac{\rho_{1}-\rho_{2}}{2} \widehat{\phi}^{n+1}+\frac{\rho_{1}+\rho_{2}}{2}, \quad \mu^{n+1}=\frac{\mu_{1}-\mu_{2}}{2} \widehat{\phi}^{n+1}+\frac{\mu_{1}+\mu_{2}}{2} ;
$$

and

$$
\begin{aligned}
& \Delta \psi^{n+1}=\frac{3 \rho_{1}}{2 \delta t} \nabla \cdot u^{n+1}, \\
& \left.\partial_{n} \psi^{n+1}\right|_{\partial \Omega}=0, \\
& p^{n+1}=p^{n}+\psi^{n+1}-\mu^{n+1} \nabla \cdot u^{n+1} .
\end{aligned}
$$

\section{Numerical Results and Discussions}

We present in this section some numerical experiments using the schemes constructed in the last section.

Let us first describe briefly our spatial discretization which is based on the Legendre-Galerkin method (cf. [21]). We use the inf-sup stable $\left(P_{N}, P_{N-2}\right)$ pair for the velocity and pressure (or the gauge or pseudo-pressure), and $P_{N}$ for the phase function $\phi$ and the chemical potential $w$.

The time discretization schemes constructed in the last section all lead to a weakly coupled system for the velocity, the phase function and the chemical potential, and an elliptic equation for the pressure. In order to take full advantage of the fast spectral Poisson solvers, we decouple the weakly coupled system by using a lagged velocity for the convective term in the equation for the phase function. Therefore, at each time step, we need to solve an elliptic equation for the velocity $u$, a system of two elliptic equations for $(\phi, w)$, and an elliptic equation or a Poisson equation (depending on which scheme to use) for the pressure $p$. The non-constant coefficient elliptic problems will be solved by using a preconditioned conjugate gradient (PCG) method with a suitable constant-coefficient problem as preconditioner (cf. [21]).

\subsection{Example 1: a lighter bubble rising in a heavier medium}

We consider the situation where a lighter bubble (with density $\rho_{1}$ and dynamic viscosity $\mu_{1}$ ) initially inside a heavier medium (with density $\rho_{2}$ and dynamic viscosity $\mu_{2}$ ) confined in a rectangular domain $\Omega=(0, \mathrm{~d}) \times\left(0, \frac{3}{2} \mathrm{~d}\right)$. 
The equations are non-dimensionalized by using the following scaled variables:

$$
\widehat{t}=\frac{t}{t_{0}}, \quad \widehat{\rho}=\frac{\rho}{\rho_{0}}, \quad \widehat{x}=\frac{x}{d_{0}}, \quad \widehat{u}=\frac{u}{u_{0}},
$$

where

$$
t_{0}=\sqrt{\frac{\mathrm{d}}{\mathrm{g}}}, \quad d_{0}=\mathrm{d} ; \quad u_{0}=\sqrt{\mathrm{dg}}, \quad \rho_{0}=\min \left(\rho_{1}, \rho_{2}\right) .
$$

The dimensionless form of (3.8) with an extra gravitational force $\rho \mathrm{g}$ in the momentum equation, after we omit the ${ }^{\wedge}$ from the notation, is

$$
\begin{aligned}
& \phi_{t}+(u \cdot \nabla) \phi+\gamma w=0 \\
& w-\Delta \phi+\frac{\phi\left(\phi^{2}-1\right)}{\eta^{2}}=0 \\
& \sigma(\sigma u)_{t}+\left(\sigma^{2} u \cdot \nabla\right) u+\frac{1}{2} \nabla \cdot\left(\sigma^{2} u\right) u-\nabla \cdot(\mu \nabla u)+\nabla p+\lambda w \nabla \phi=\rho \mathrm{g}, \\
& \nabla \cdot u=0
\end{aligned}
$$

with

$$
\rho(\phi)=\frac{\widetilde{\rho}_{1}-\widetilde{\rho}_{2}}{2} \phi+\frac{\widetilde{\rho}_{1}+\widetilde{\rho}_{2}}{2}, \quad \sigma(\phi)=\sqrt{\rho(\phi)}, \quad \mu(\phi)=\frac{\widetilde{\mu}_{1}-\widetilde{\mu}_{2}}{2} \phi+\frac{\widetilde{\mu}_{1}+\widetilde{\mu}_{2}}{2} .
$$

In the above, $\widetilde{\rho}_{1}=\frac{\rho_{1}}{\rho_{0}}, \widetilde{\rho}_{2}=\frac{\rho_{2}}{\rho_{0}}, \widetilde{\mu}_{1}=\frac{\mu_{1}}{\left(\rho_{0} \mathrm{~d}^{\frac{3}{2}} \mathrm{~g}^{\frac{1}{2}}\right)}, \widetilde{\mu}_{2}=\frac{\mu_{2}}{\left(\rho_{0} \mathrm{~d}^{\frac{3}{2}} \mathrm{~g}^{\frac{1}{2}}\right)}$.

We set the initial velocity to be zero and initial phase function given by

$$
\phi(x, t=0)=-\tanh \left(\frac{\mathrm{r}-\frac{1}{4} \mathrm{~d}}{\eta_{0}}\right),
$$

where $\mathrm{r}$ is the distance from the center of the bubble to the point and $\eta_{0}$ is the diffusive interfacial width.

We consider first the case where $\rho_{1}$ and $\rho_{2}$ are not too far apart that we can use the following Boussinesq approximation for (4.3):

$$
\begin{aligned}
& \phi_{t}+u \cdot \nabla \phi+\gamma \Delta w=0, \\
& w-\Delta \phi+f(\phi)=0, \\
& \rho_{0}\left(u_{t}+(u \cdot \nabla) u+\nabla p-\nabla \cdot(\mu D(u))+\lambda w \nabla \phi\right)=-g\left(2 \rho_{0}+\rho_{1}+\rho_{2}\right)-g \phi\left(\rho_{1}-\rho_{2}\right), \\
& \nabla \cdot u=0 .
\end{aligned}
$$

In the first example, we set $\rho_{0}=1, \rho_{1}-\rho_{2}=-1$ and $g=(10,0)^{t}, \mu=1, \lambda=0.001, \gamma=2 \times 10^{-8}$ and $\eta_{0}=\eta=0.02 \mathrm{~d}$. In Figure 1, we plot the interface contour of $\{\phi: \phi(x)=0\}$ at several different times obtained by using the scheme (3.9) with a grid size of $257^{2}$. These results are qualitatively consistent with the results presented in [23] where the Allen-Cahn, instead of the Cahn-Hilliard, phase-field model is used. Note that while both the Allen-Cahn and CahnHilliard phase field models are legitimate approximations to the sharp interface model, their transient histories (with fixed relaxation constants $\gamma$ ) can differ due to the different relaxation time scales in the two models. 

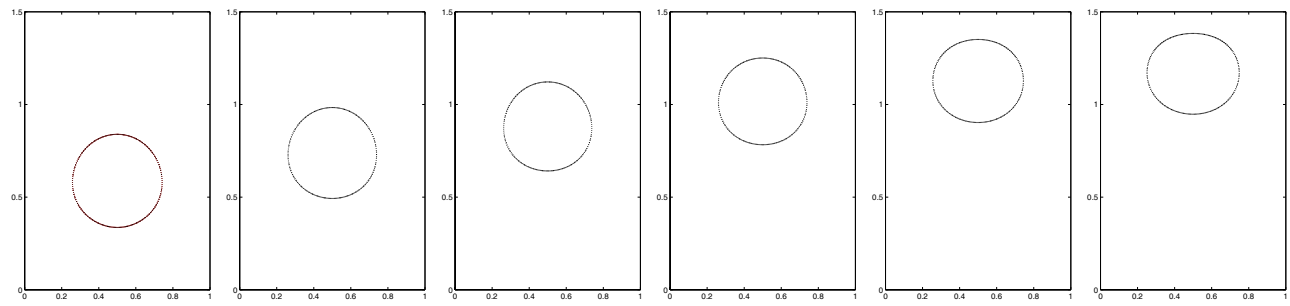

Figure 1 Example 1: snapshots of interfaces contours of $\phi$ at $t=1,3,5,7,9,10$.

\subsection{Example 2: an air bubble rising in water}

The second example we consider is an air bubble rising in water. The physical parameters are $\rho_{1}=1.161$ and $\rho_{2}=995.65$ with $\mu_{1}=0.0000186, \mu_{2}=0.0007977$. We set $\mathrm{d}=0.005, \mathrm{~g}=$ $9.8, \lambda=0.001, \gamma=2 \times 10^{-8}$ and $\eta_{0}=\eta=0.02 \mathrm{~d}$. In this case, the Boussinesq approximation is no longer valid so we solve the system (4.3) directly by using the schemes (3.19) and (3.28). We use a grid size of $257^{2}$ and time step size of $\delta t=0.0001$.
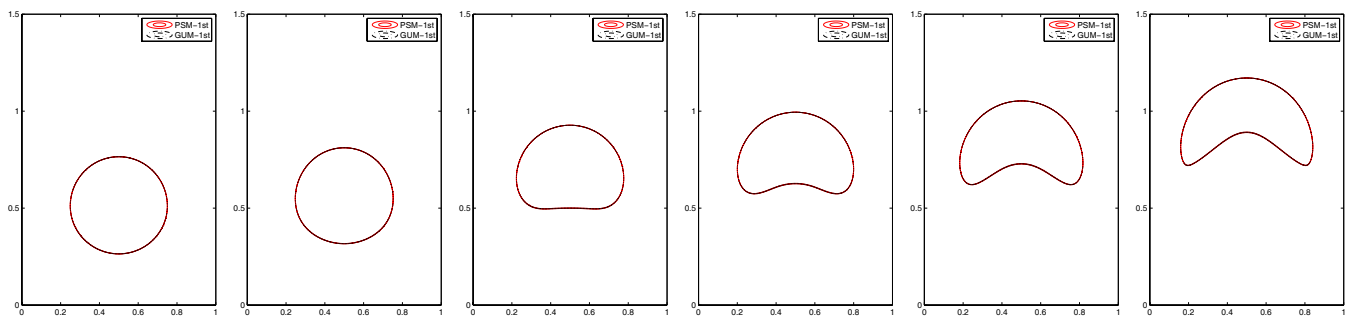

(a) Interfaces at different time snapshots $t=0.5,1,1.5,2,2.5,3$.

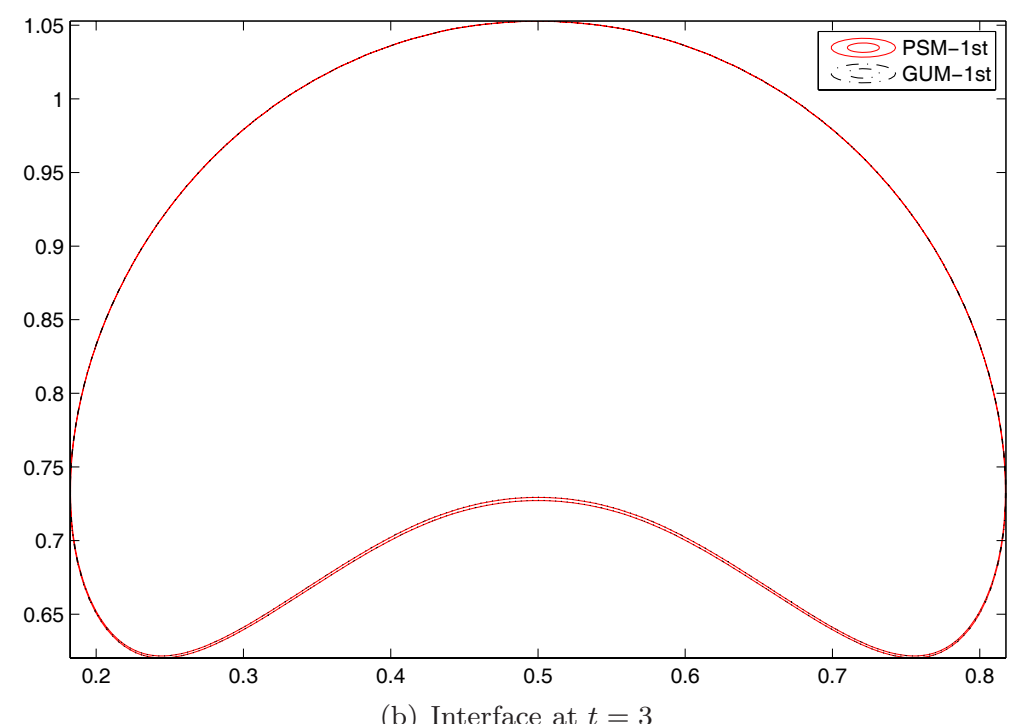

Figure 2 Example 2: snapshots at $t=0.5,1,1.5,2,2.5,3$.

In Figure 2, we plot a comparison of the level sets $\{\phi: \phi=0\}$ by the two schemes at different times. We observe that the two schemes produce visually identical results. Once again, the results are qualitatively similar to those in [23] produced by a Allen-Cahn phase-field model. 


\subsection{Summary}

We considered the numerical approximation of Cahn-Hilliard phase-field model for the twophase incompressible flows in this paper.

We constructed several efficient and energy stable time discretization schemes for the coupled nonlinear Cahn-Hilliard phase-field system for both the matched density case and the variable density case. At each time step, these schemes all reduce to a weakly coupled system for the velocity $u$, phase function $\phi$ and chemical potential $w$, and an elliptic or Poisson equation for the pressure $p$. Thus, existing elliptic solvers can be efficiently adopted.

We also carried out numerical experiments which validated the Cahn-Hilliard phase-field model with matched and variable densities and the proposed numerical schemes.

We note that while we have only considered the semi-discretization in time, the proofs are all based on a variational formualtion, so they can be in principle extended to consistent full discretizations with Galerkin finite elements or Galerkin-spectral methods.

\section{References}

[1] Allen, S. M. and Cahn, J. W., A microscopic theory for antiphase boundary motion and its application to antiphase domain coarsening. Acta Metall. Mater., 27, 1979, 1085-1095.

[2] Anderson, D. M., McFadden, G. B. and Wheeler, A. A., Diffuse-interface methods in fluid mechanics, 30, 1998, 139-165.

[3] Becker, R., Feng, X. and Prohl, A., Finite element approximations of the Ericksen-Leslie model for nematic liquid crystal flow, SIAM J. Numer. Anal., 46(4), 2008, 1704-1731.

[4] Caffarelli, L. A. and Muler, N. E., An $L^{\infty}$ bound for solutions of the Cahn-Hilliard equation, Arch. Rational Mech. Anal., 133(2), 1995, 129-144.

[5] Cahn, J. W. and Hilliard, J. E., Free energy of a nonuniform system, I: Interfacial free energy, J. Chem. Phys., 28, 1958, 258-267.

[6] Condette, N., Melcher, C. and Süli, E., Spectral approximation of pattern-forming nonlinear evolution equations with double-well potentials of quadratic growth, Math. Comp., to appear.

[7] Feng, X., He, Y. and Liu, C., Analysis of finite element approximations of a phase field model for two-phase fluids (electronic), Math. Comp., 76(258), 2007, 539-571.

[8] Guermond, J. L., Minev, P. and Shen, J., An overview of projection methods for incompressible flows, Comput. Methods Appl. Mech. Engrg., 195, 2006, 6011-6045.

[9] Guermond, J. L. and Quartapelle, L., A projection FEM for variable density incompressible flows, J. Comput. Phys., 165(1), 2000, 167-188.

[10] Guermond, J. L. and Salgado, A., A splitting method for incompressible flows with variable density based on a pressure Poisson equation, J. Comput. Phys., 228(8), 2009, 2834-2846.

[11] Gurtin, M. E., Polignone, D. and Vinals, J., Two-phase binary fluids and immiscible fluids described by an order parameter, Math. Models Methods Appl. Sci., 6(6), 1996, 815-831.

[12] Jacqmin, D., Diffuse interface model for incompressible two-phase flows with large density ratios, J. Comput. Phys., 155(1), 2007, 96-127.

[13] Kessler, D., Nochetto, R. H. and Schmidt, A., A posteriori error control for the Allen-Cahn problem: circumventing Gronwall's inequality, M2AN Math. Model. Numer. Anal., 38(1), 2004, 129-142.

[14] Lin, P., Liu, C. and Zhang, H., An energy law preserving $C^{0}$ finite element scheme for simulating the kinematic effects in liquid crystal dynamics, J. Comput. Phys., 227(2), 2007, 1411-1427.

[15] Liu, C. and Shen, J., A phase field model for the mixture of two incompressible fluids and its approximation by a Fourier-spectral method, Physica D, 179(3-4), 2003, 211-228.

[16] Lowengrub, J. and Truskinovsky, L., Quasi-incompressible Cahn-Hilliard fluids and topological transitions, R. Soc. Lond. Proc., Ser. A, Math. Phys. Eng. Sci., 454(1978), 1998, 2617-2654.

[17] Nochetto, R. and Pyo, J. H., The gauge-Uzawa finite element method part I: the Navier-Stokes equations, SIAM J. Numer. Anal., 43, 2005, 1043-1068. 
[18] Prohl, A., Projection and quasi-compressibility methods for solving the incompressible Navier-Stokes equations, Advances in Numerical Mathematics, BG Teubner, Stuttgart, 1997.

[19] Pyo, J. and Shen, J., Gauge-uzawa methods for incompressible flows with variable density, J. Comput. Phys., 221, 2007, 181-197.

[20] Rannacher, R., On Chorin's projection method for the incompressible Navier-Stokes equations, Lecture Notes in Mathematics, 1530, Springer-Verlag, Berlin, 1991.

[21] Shen, J., Efficient spectral-Galerkin method I. direct solvers for second- and fourth-order equations by using Legendre polynomials, SIAM J. Sci. Comput., 15, 1994, 1489-1505.

[22] Shen, J., On error estimates of projection methods for the Navier-Stokes equations: second-order schemes, Math. Comp, 65, 1996, 1039-1065.

[23] Shen, J. and Yang, X., A phase-field model and its numerical approximation for two-phase incompressible flows with different densities and viscosities, SIAM J. Sci. Comput., 32, 2010, 1159-1179.

[24] Shen, J. and Yang, X., Numerical approximations of allen-cahn and cahn-hilliard equations, Discrete and Continuous Dynamical Systems, Series A, 28, 2010, 1669-1691.

[25] Walkington, N. J., Compactness properties of the DG and CG time stepping schemes for parabolic equations, SIAM J. Numer. Anal., 47(6), 2010, 4680-4710.

[26] Yue, P., Feng, J. J., Liu, C., et al., A diffuse-interface method for simulating two-phase flows of complex fluids, J. Fluid Mech, 515, 2004, 293-317. 